\title{
Liaison meetings between a psychiatric team and general practitioners: description and evaluation of a pilot project
}

\author{
David Westbrook, Principal Clinical Psychologist, Department of Clinical \\ Psychology, and KeITH Hawton, Consultant Psychiatrist and Clinical Lecturer, \\ Warneford Hospital, Headington, Oxford OX3 7JX
}

For more than 20 years, in parallel with moves towards community care, psychiatric teams have been trying to develop closer links with general practice. Many styles of working with GPs have been developed, five of which are described by Mitchell (1989). The literature contains numerous reports of most of these, particularly the shifted out-patient model (see for example Tyrer, Seivewright \& Wollerton, 1984). In this paper we describe a liaison project which is closest to Mitchell's (1989) 'joint team consultation' and report the results of a simple survey of GP satisfaction with this scheme.

\section{The study}

\section{Aims and approach}

The psychiatric services in Oxfordshire have recently been sectorised, with each acute team taking responsibility for an area of the county. In planning this project, the psychiatric team for West Oxfordshire had the broad aims of improving communications with local GPs and providing support and advice for their management of patients with psychological problems.

The scheme chosen was closest to Mitchell's (1989) third model, 'joint team consultation', since it was felt that this offered the best combination of direct patient discussion with more indirect mutual education.

Four groups of GPs were included in the pilot project. The psychiatric team consisted of a clinical psychologist, a senior registrar, and a community psychiatric nurse. The consultant psychiatrist attended one meeting at each practice. A typical meeting consisted of the three members of the psychiatric team and between four and six GPs, and the initial pilot project ran for six monthly meetings at each practice.

\section{Evaluation procedure}

Six topics which had consistently been ranked as most important in a previous survey were taken as the focus of liaison discussions and of the evaluation feedback. The areas chosen were: assessment of psychiatric problems; pharmacological management; psychological management; difficulties GPs encountered with psychiatric services; appropriate referral of psychological problems; and improvement of communication.

After the six meetings with each group, GPs were asked to rate each of these aspects of the meetings on a series of 7-point scales to assess: whether too much or too little time was spent on that topic; how useful each area of discussion had been; and how much the meetings had influenced their management of specific patients discussed and their general management of patients with psychological problems.

In addition, GPs were asked for their views on the composition of the psychiatric team and whether they would attend further liaison meetings.

\section{Evaluation findings}

The total number of GPs completing feedback questionnaires was 22 . The overall mean ratings of 'time spent' indicated little difference between different topic areas, all getting ratings just below 'about right'.

The areas receiving highest 'usefulness' ratings were 'assessment', 'psychological management' and 'communication'. Two groups of GPs rated most aspects of the meetings as less useful than did the other two groups. Groups' ratings of the overall usefulness of the meetings ranged from 1.5 to 4.8 , with an overall mean of 3.7 , or just above 'moderately useful'.

On the 'influence' ratings, overall means were just above 'moderate', but this again masked considerable variation between practices, with the two groups with lower 'usefulness' ratings also rating lower on this scale.

Regarding the composition of the team, many GPs thought the membership was perfectly acceptable, but some doctors from three of the four groups suggested that the consultant should also attend more frequently. Reasons were not always given, but included views that the consultant should attend as 
"the legally responsible person" and that it would be easier to discuss service deficiencies if he were there.

Sixteen GPs said they would continue to attend meetings in the present format, while six said they would continue only if the meetings changed in various ways. The most common requested were more frequent attendance of the consultant, and more structured meetings, with more preparation of cases beforehand.

\section{Comments}

The liaison meetings appear to have been quite successful and meetings have continued with most of the practices since the pilot project ended. The time and usefulness ratings suggest that the balance of topics was generally about right, though two of the practices seem to have been less satisfied (a possible explanation is that these two groups were concerned about the recent loss of an out-patient psychiatric clinic which used to be held in their town).

The most popular aspect of the meetings seemed to be the chance to forge closer relationships between GPs and the psychiatric team. Of the more specific topics, discussion of assessment and psychological management seemed to be the most valued. Nearly three-quarters of the GPs said that they would continue attending meetings in their present form.

Long-term continuation of this scheme is likely to necessitate some changes, as the importance of GPs and the psychiatric team getting to know each other will diminish as relationships are strengthened. Options identified either by ourselves or by GPs include, first, that (as several GPs felt) the meetings could be more structured; and second, that GPs and psychiatric team members might work together on particular cases, either by seeing patients for joint assessments, as in the 'tripartite' model of Mitchell (1979), or by interested GPs having more intensive training or supervision in psychological management of their cases.

\section{Acknowledgements}

We would like to thank the GPs in West Oxfordshire who took part in this project, all the other members of the visiting psychiatric team (Anita Damle, Catherine Gardiner, Roy Overall, Rob Poole, Bill Trotter and Jane Webb), and Joan Kirk for her comments on an earlier version of the manuscript.

\section{References}

Mrtchell, A. R. K. (1989) Participating in primary care: differing styles of psychiatric liaison. Psychiatric Bulletin, 13, 135-137.

Tyrer, P., Seivewright, N. \& Wollerton, S. (1984) General practice psychiatric clinics: impact on psychiatric services. British Journal of Psychiatry, 145, 15-19.

\title{
Self-referral admissions
}

\author{
Martin Gee, Senior Registrar in Psychiatry, Substance Abuse Unit (Ward 93), City \\ General Hospital, Newcastle Road, Stoke-on-Trent ST4 6QG; and NiCK CRADDOCK, \\ Sheldon Research Fellow, Department of Psychiatry, University of Birmingham, \\ Queen Elizabeth Hospital, Birmingham B15 2TH
}

Little is known about those patients who bypass their GP and self-refer directly to hospital. There have been studies of referrals to emergency clinics (Lim, 1983; Haw et al, 1987; Kehoe \& Newton, 1990) and to community centres (Hutton, 1983; Boardman \& Bouras, 1989). All these studies found that more men self-referred than women. Hutton (1985), Boardman \& Bouras (1989) and Kehoe \& Newton (1990) found that self-referrals often had a non-psychiatric precipitant and rarely required acute psychiatric intervention.

The process of self-referral to hospital differs from self-referral to community units or emergency clinics in that patients are usually discouraged from the former but encouraged in the latter.

We conducted the current study to establish the features of patients who self-refer to psychiatric hospital and to identify any differences between such 